\title{
The Changing Nature of Work: From Structured to Unstructured, from Controlled to Social
}

\author{
Sandy Kemsley \\ Kemsley Design Ltd., Toronto , Canada \\ sandy@kemsleydesign.com
}

\begin{abstract}
Traditional BPM systems perform well with structured processes and controlled interactions between participants. However, most business functions involve both structured processes with ad hoc activities, and can range from topdown delegation to social collaboration. A key trend in business is the increased focus on knowledge worker productivity as the routine work becomes more automated, which has led to fragmentation of the BPM market: in addition to traditional BPM systems, a new breed of adaptive case management (ACM) systems manage unstructured processes, collaboration is taking hold both in process design and runtime environments, and user interfaces are borrowing from social media to put a new face on BPM.

But do the differences between structured and unstructured process management, or between authoritarian and collaborative interactions, warrant the use of different types of systems? Or do they just represent different parts of a spectrum of process functionality? How can they best be combined to manage a broad variety of business process types? What types of hybrid process systems will emerge to match these business needs?
\end{abstract}

